\title{
Dynamics of a two-dimensional vapor bubble confined between superheated or subcooled parallel plates
}

\author{
Kausik S. Das \\ Department of Mechanical and Industrial Engineering, University of Toronto, 5 King's College Road, Toronto, \\ Ontario, Canada M5S $3 G 8$ \\ Stephen K. Wilson* \\ Department of Mathematics and Statistics, University of Strathclyde, Livingstone Tower, 26 Richmond Street, \\ Glasgow G1 1XH, United Kingdom
}

(Received 3 June 2009; revised manuscript received 27 January 2010; published 22 April 2010)

\begin{abstract}
The dynamics of a long, two-dimensional vapor bubble confined in the gap between two superheated or subcooled parallel plates is analyzed theoretically. The unsteady expansion and/or contraction of the bubble is driven by mass transfer between the liquid and the vapor. The analysis uses the approach developed by Wilson et al. [J. Fluid Mech. 391, 1 (1999)] for a situation with "large" gaps and "small" superheating or subcooling to consider a situation with small gaps and large superheating or subcooling in which the mass transfer from or to the semicircular nose of the bubble is comparable to that from or to the thin liquid films on the plates. In order to permit a (semi-) analytical treatment the analysis is restricted to low Prandtl number liquids. When both plates are superheated the bubble always expands. In this case there are two possible constant-velocity continuous-film solutions for the expansion of the bubble, namely, an unstable fast mode and a stable slow mode. The evolution of the bubble is calculated numerically for a range of values of the parameters. In particular, these calculations show that eventually the bubble expands either with the constant velocity of the slow mode or exponentially. When both plates are subcooled the bubble always collapses to zero length in a finite time. When one plate is subcooled and the other plate is superheated the situation is rather more complicated. If the magnitude of the subcooling is less than that of the superheating then if the magnitude of the subcooling is greater than a critical value then a variety of complicated behaviors (including the possibility of an unexpected "waiting time" behavior in which the bubble remains almost stationary for a finite period of time) can occur before the bubble eventually collapses to a finite length in an infinite time, whereas if it is less than this critical value then the bubble always expands and eventually does so exponentially. If the magnitude of the subcooling is greater than that of the superheating then the bubble always collapses to zero length in a finite time.
\end{abstract}

DOI: 10.1103/PhysRevE.81.046314

PACS number(s): 47.55.dd, 47.55.dp

\section{INTRODUCTION}

As a consequence of its applications in a wide range of areas including aerospace science, microelectromechanical systems (MEMS), compact heat exchangers, process intensification and chemical microreactors, there is currently considerable research activity on microscale nucleate boiling. In both MEMS and microactuators, geometrically constrained vapor bubbles are used to move mechanical parts and to pump liquid in microchannels by localized heating. In a bubble jet printer, the ejection of ink is controlled by the expansion and contraction of vapor bubbles. Flow boiling in narrow channels is also used to generate vapor bubbles in various refrigeration and power systems, and in cooling systems such as chemical reactors in which intensive heat generation takes place. Heat generating porous materials in which vapor bubbles enter heated capillaries are also of interest because of their relevance to the radioactive debris caused by a serious nuclear accident.

Wilson, Davis and Bankoff [1] (hereafter referred to as "WDB" for brevity) studied the dynamics of a long, two-

\footnotetext{
*Corresponding author. FAX: + 44 (0)141 5528657. s.k.wilson@strath.ac.uk
}

dimensional vapor bubble confined in the gap between two equally superheated or subcooled parallel plates. Unlike Bretherton's [2] classical isothermal problem, in which the steady translation of the bubble is driven by an externally imposed pressure gradient, they studied the unsteady expansion and/or contraction of a vapor bubble whose motion is driven by mass transfer between the liquid and the vapor. As in Bretherton's problem, the velocity of the bubble determines the initial thickness of the thin films of liquid laid down on both plates as the bubble expands, but unlike in Bretherton's problem the evaporation from and/or condensation onto those films (which may break up into disconnected patches of liquid as they evaporate) determines the velocity of expansion and/or contraction of the bubble, and so there is a nonlinear coupling with a delay character between the profiles of the thin films and the overall dynamics of the bubble. WDB investigated a particular parameter regime in which mass transfer between the liquid and the vapor was dominated by that from or to the thin liquid films on the plates. In the present work we shall use the approach developed by WDB to consider an alternative parameter regime in which the mass transfer from or to the semicircular nose of the bubble is comparable to that from or to the thin films. 
Ajaev and Homsy $[3,4]$ studied a steady vapor bubble in a rectangular channel with a prescribed temperature distribution on its walls in which there is a balance between evaporation from the hotter parts of the bubble interface and condensation onto the colder parts, while Ajaev et al. [5] considered a steady two-dimensional vapor bubble between two parallel plates held at different temperatures, and then investigated its dynamic response to temporally varying plate temperatures. A review of the mathematical models for both the steady shapes and dynamics of confined vapor bubbles has recently been given by Ajaev and Homsy [6].

In practice, there is often a net flow along the channel and so numerical simulations of the unsteady growth of a vapor bubble in a rectangular channel with prescribed temperatures on its walls in this so-called "flow boiling" regime were obtained by Mukherjee and Kandlikar [7], while experimental measurements of flow boiling in a partially heated channel were recently reported by Wang and Cheng [8].

Helpful overviews of the rapidly growing literature on boiling and two-phase flow in microchannels have been given by Kandlikar [9], Thome [10], and in the introduction to the work by Kenning et al. [11].

\section{PROBLEM FORMULATION}

Following WDB we consider a long, two-dimensional vapor bubble of inviscid and incompressible vapor with density $\rho^{(V)}$ surrounded by its condensate and confined between two parallel plates a distance $2 d$ apart. The condensate is assumed to be a Newtonian liquid with constant viscosity $\mu$, kinematic viscosity $\nu$, density $\rho$, surface tension $\sigma$, thermal diffusivity $\kappa$, and thermal conductivity $k$. The effect of gravity is neglected. The two plates (hereafter referred to as the "upper" and "lower" plates for simplicity) are held at (in general different) uniform temperatures $T_{\mathrm{u}}$ and $T_{1}$, respectively, which may be either above or below the saturation temperature $T_{\mathrm{s}}$.

We choose Cartesian coordinates $(x, y)$ so that the parallel plates are at $y= \pm d$, and consider only bubbles that are symmetric about the $y$ axis. For simplicity of presentation in much of what follows we shall confine our attention to the lower quarter of the bubble lying in $x>0$ and $y<0$; the corresponding results in the upper quarter of the bubble lying in $x>0$ and $y>0$ can be readily deduced. For convenience, we write $T_{\mathrm{u}}=T_{\mathrm{s}}+\Delta T_{\mathrm{u}}$ and $T_{1}=T_{\mathrm{s}}+\Delta T_{1}$ and consider the three specific situations sketched in Fig. 1, namely, (a) both plates superheated, $\Delta T_{\mathrm{u}}, \Delta T_{1}>0$, (b) both plates subcooled, $\Delta T_{\mathrm{u}}, \Delta T_{1}<0$, and (c) the upper plate subcooled and the lower plate superheated, $\Delta T_{\mathrm{u}}<0$ and $\Delta T_{1}>0$.

We nondimensionalize length with $d$, velocity with $\nu / d$, time with $d^{2} / \nu$, pressure with $\sigma / d$, and temperature difference from the saturation temperature with $\left|\Delta T_{1}\right|$. The energy balance at the interface gives the mass flux scale as $k\left|\Delta T_{1}\right| / \mathcal{L} d$, where $\mathcal{L}$ is the latent heat of vaporization. Hereafter, all quantities will be dimensionless unless stated otherwise. In what follows we shall retain $\Delta T_{1}$ for clarity of presentation, although the choice of nondimensionalization means that it can be set equal to either plus or minus unity without loss of generality.
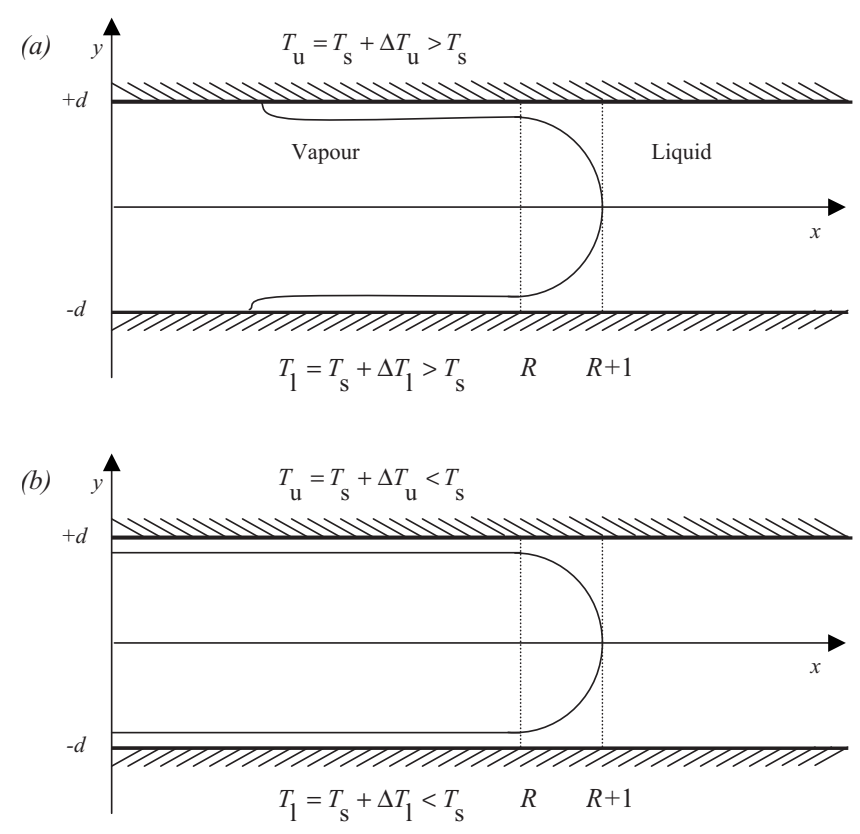

(c)

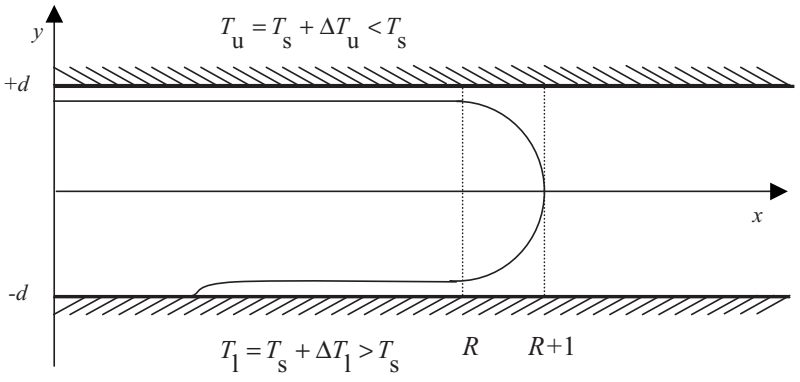

FIG. 1. The geometry of the three problems studied: (a) both plates superheated, (b) both plates subcooled and (c) the upper plate subcooled and the lower plate superheated.

The velocity $(u, v)$, where $u=u(x, y, t)$ and $v=v(x, y, t)$, pressure $p=p(x, y, t)$ and temperature $T=T(x, y, t)$ of the liquid satisfy the usual continuity, Navier-Stokes and energy equations,

$$
\begin{gathered}
u_{x}+v_{y}=0, \\
C\left(u_{t}+u u_{x}+v u_{y}\right)=-p_{x}+C\left(u_{x x}+u_{y y}\right), \\
C\left(v_{t}+u v_{x}+v v_{y}\right)=-p_{y}+C\left(v_{x x}+v_{y y}\right), \\
P\left(T_{t}+u T_{x}+v T_{y}\right)=T_{x x}+T_{y y},
\end{gathered}
$$

where $C=\rho \nu^{2} / \sigma d$ is the capillary number, $P=\nu / \kappa$ is the Prandtl number, and $t$ denotes time. Similar equations hold in the vapor. However, since the ratios of density, viscosity and thermal conductivity of the vapor to those of the liquid are typically small, we shall neglect all dynamic processes in the vapor. In the spirit of the classical Boussinesq approximation, the vapor density is retained only where it multiplies the large vapor velocity. This so-called "one-sided" model developed by Burelbach et al. [12] allows us to obtain simple boundary conditions for the liquid without solving for the flow and heat transfer in the vapor. 
At the lower liquid-vapor interface $y=-1+h(x, t)$ the local mass-balance condition gives

$$
-E J=\left(h_{t}+u h_{x}-v\right)\left(1+h_{x}^{2}\right)^{-1 / 2},
$$

where $J=J(x, t)$ denotes the mass flux at the interface due to evaporation or condensation and $E=k\left|\Delta T_{1}\right| / \mathcal{L} \rho \nu$ is the nondimensional evaporation number. The local energy-balance condition at the interface yields

$$
J=\left(T_{x} h_{x}-T_{y}\right)\left(1+h_{x}^{2}\right)^{-1 / 2},
$$

while the normal-stress condition is

$$
\begin{aligned}
-p & +2 C\left[v_{y}+h_{x}^{2} u_{x}-h_{x}\left(u_{y}+v_{x}\right)\right]\left(1+h_{x}^{2}\right)^{-1} \\
& =h_{x x}\left(1+h_{x}^{2}\right)^{-3 / 2},
\end{aligned}
$$

and the tangential-stress condition can be written as

$$
\left(1-h_{x}^{2}\right)\left(u_{y}+v_{x}\right)-2 h_{x}\left(u_{x}-v_{y}\right)=0 \text {. }
$$

The boundary conditions at the plates $y= \pm 1$ are no slip, $u$ $=v=0$, and prescribed temperature, $T=\Delta T_{\mathrm{u}}$ at the upper plate $y=1$ and $T=\Delta T_{1}$ at the lower plate $y=-1$.

One further (constitutive) equation is required in order to close the system, and this is taken to be a linearized version of the nonequilibrium condition relating the interfacial mass flux to the interfacial temperature

$$
K J=T,
$$

where

$$
K=\frac{k T_{\mathrm{s}}^{3 / 2}}{\alpha \mathcal{L}^{2} \rho^{(V)} d}\left(\frac{2 \pi R_{\mathrm{g}}}{M_{\mathrm{w}}}\right)^{1 / 2}
$$

is a nondimensional kinetic parameter which measures the degree of nonequilibrium at the interface, with $R_{\mathrm{g}}=8.31 \mathrm{~J} \mathrm{~mol}^{-1} \mathrm{~K}^{-1}$ denoting the gas constant, $M_{\mathrm{w}}$ the molecular weight of the vapor, and $\alpha$ the accommodation coefficient (see, for example, Burelbach et al. [12], the more detailed discussion by Panzarella [13], and the extensive review of literature concerning the evaporation and condensation coefficients of water by Marek and Straub [14]).

Finally, global conservation of mass of liquid and vapor means that the rate of change of the mass of the bubble is equal to the total mass flux into the bubble and so

$$
2 U=D E \int J d s,
$$

where $U=U(t)$ is the velocity of the bubble, $D=\rho / \rho^{(V)}$ is the ratio of the liquid density to vapor density, and the integral is over the entire liquid-vapor interface in $x>0$, parameterized by its arclength $s$.

\section{ASYMPTOTIC SOLUTION IN THE LIMIT $C \rightarrow 0$}

In the present work we shall follow Bretherton [2], WDB, and many other previous authors by considering the limit of strong surface tension (i.e., the limit of small capillary number $C \rightarrow 0$ ) in which the bubble almost entirely fills the gap between the plates and an expanding bubble leaves behind thin liquid films on the plates as it grows. In this limit the solution is composed of three different kinds of region. Specifically, the asymptotic solution in $x>0$ consists of a "capillary-statics" region occupying the gap between the plates when $R(t)<x<R(t)+1$ in which capillary effects dominate and the leading-order solution for the liquid-vapor interface is a semicircular cap of radius unity that fits exactly between the plates, two small and thin "transition" regions of length $O\left(C^{1 / 3}\right) \ll 1$ and thickness $O\left(C^{2 / 3}\right) \ll 1$ near $x=R(t)$ and $y= \pm d$ (i.e., where the semicircular cap touches the plates) in which viscous effects become significant and (in the case of an expanding bubble) thin films of thickness $O\left(C^{2 / 3}\right) \ll 1$ are laid down on the plates, and two "thin-film" regions occupying $0<x<R(t)$ in which there are thin films of thickness $O\left(C^{2 / 3}\right) \ll 1$ on the plates. The (unknown) length of the thin-film regions $R(t)$ is calculated from the (unknown) velocity of the bubble $U(t)$ which depends on the total mass flux according to Eq. (11).

In their analysis WDB investigated a parameter regime in which $D=O\left(C^{-2 / 3}\right) \gg 1, E=O\left(C^{4 / 3}\right) \ll 1$, and $K=O\left(C^{2 / 3}\right) \ll 1$. In this situation the leading order total mass flux is determined solely by the mass flux from or to the thin-film regions, and the higher order contributions to the mass flux from the capillary-statics and transition regions can be neglected at leading order. In the present work we investigate an alternative parameter regime in which $D=O\left(C^{-2 / 3}\right) \gg 1$ as before but $E=O\left(C^{2 / 3}\right) \ll 1$ and $K=O(1)$. In this situation the mass fluxes from the capillary-statics region and the thinfilm regions both contribute to the leading order total mass flux, and only the higher order contribution from the transition regions can be neglected at leading order. For clarity, we write $D=\hat{D} C^{-2 / 3}$ and $E=\hat{E} C^{2 / 3}$, where $\hat{D}$ and $\hat{E}$ are both $O(1)$ in the limit $C \rightarrow 0$.

\section{A. Capillary-statics region}

The calculation of the mass flux from the capillary-statics region involves determining the temperature distribution in the liquid in $x>R$ by solving the energy Eq. (4) subject to the boundary conditions (5) and (6) at the (known) liquidvapor interface and prescribed temperature on the plates. In general, this is a difficult numerical problem involving solving for both $u$ and $T$ in a reference frame moving with unknown velocity $U(t)$ given by Eq. (11). We can, however, make progress in the special case of small Prandtl number $P=o(1)$ (i.e., when thermal diffusion is much stronger than viscous diffusion) in which case the problem for $T$ decouples from that for $u$ and depends on only $\Delta T_{\mathrm{u}}, \Delta T_{1}$ and $K$ (and not, for example, on $D$ or $E$ ). In this case the contribution to the integral in Eq. (11) from the capillary-statics region is given by $\left(\Delta T_{\mathrm{u}}+\Delta T_{1}\right) S(K)$, where straightforward numerical calculations (undertaken using a standard finite element method implemented using the software package COMSOL Multiphysics, formerly FEMLAB [15]) reveal that the coefficient $S=S(K)$ is a monotonically decreasing function of $K$ satisfying $S=O\left(K^{-1 / 2}\right) \rightarrow \infty$ as $K \rightarrow 0^{+}, S(1)=1.2391$, and $S=O\left(K^{-1}\right) \rightarrow 0$ as $K \rightarrow \infty$. Figure 2 shows numerically calculated values of $S(K)$ plotted as a function of $K$. While the assumption that $P=o(1)$ permits the present (semi-) analyti- 


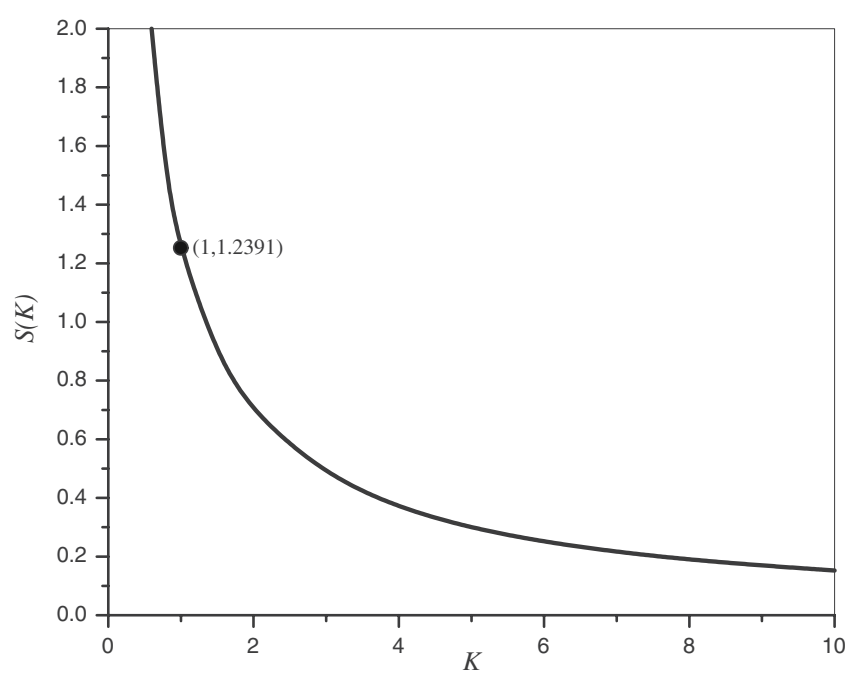
of $K$.

FIG. 2. Numerically calculated values of $S$ plotted as a function

cal treatment it does, of course, mean that the present analysis is formally restricted to low Prandtl number liquids such as the liquid and alkali metals and their alloys with values of $P$ in the range 0.005 to 0.03 which are, for example, used as coolants in nuclear reactors (see, for example, Saravanan and Kandaswamy [16]). We would, however, anticipate that some of the qualitative features described here will also occur for liquids (such as, for example, water) with larger values of $P$.

\section{B. Transition regions}

A straightforward modification of the analysis presented by WDB reveals that the leading-order solution for the liquid-vapor interface is simply the classical isothermal solution and so can be obtained in the usual way (see, for example, Bretherton [2]). In particular, the solution in the thin-film regions yields an expression for the initial thickness of the film as it is laid down on the plates when the bubble expands (i.e., when $U>0$ ), namely, $h=c U^{2 / 3} C^{2 / 3}$, where $c$ is a numerically determined constant. When the bubble retreats (i.e., when $U<0$ ) the liquid on the plates is "swept up" by the transition regions and so the details of the solution in the transition regions are unimportant.

\section{Thin-film regions}

Calculating the mass flux from the thin-film regions involves determining the temporal and spatial evolution of the profile of and the temperature distribution within the thin films on the plates. Unless they are swept up by a retreating bubble, on a superheated plate these films will thin before eventually drying out locally (possibly breaking up into disconnected patches of liquid as they do so) due to evaporation to the vapor, whereas on a subcooled plate they will always remain continuous as they thicken due to condensation from the vapor. In order to analyze this behavior, and hence determine the total mass flux, for the thin film on the lower plate (a similar analysis applies for the film on the upper plate) we introduce appropriately rescaled variables (denoted by an overbar) given by

$$
\bar{v}=C^{-2 / 3} v, \quad \bar{y}=C^{-2 / 3}(y+1), \quad \bar{h}=C^{-2 / 3} h .
$$

In the limit $C \rightarrow 0$ we obtain the leading-order lubrication equations

$$
\begin{gathered}
u_{x}+\bar{v}_{\bar{y}}=0, \\
u_{\bar{y} \bar{y}}=0, \\
0=p_{\bar{y}}, \\
T_{\bar{y} \bar{y}}=0,
\end{gathered}
$$

subject to the leading-order boundary conditions

$$
\begin{gathered}
-\hat{E} J=\bar{h}_{t}+u \bar{h}_{x}-\bar{v}, \\
0=T_{\bar{y}}, \\
p=0, \\
u_{\bar{y}}=0, \\
K J=T
\end{gathered}
$$

at the lower bubble interface $\bar{y}=\bar{h}(x, t)$, and $u=\bar{v}=0$ and $T$ $=\Delta T_{1}$ on the lower plate $\bar{y}=0$. The leading-order solution is simply $u=\bar{v}=0$ together with $T=\Delta T_{1}$ and $J=\Delta T_{1} / K$, meaning that the liquid is quiescent and at the same uniform temperature as the plate and that the flux is constant, and so Eq. (17) becomes

$$
\bar{h}_{t}+\frac{\hat{E} \Delta T_{1}}{K}=0
$$

Hence, the profile of the film in the thin-film region is given by

$$
\bar{h}(x, t)=\bar{h}_{0}(x)-\frac{\hat{E} \Delta T_{1}}{K}\left[t-t_{0}(x)\right],
$$

where if $x<L$ then $\bar{h}_{0}=\bar{h}_{0}(x)$ denotes the initial profile of the thin film at $t=t_{0}=0$, while if $x>L$ then $\bar{h}_{0}=\bar{h}_{0}(x)=c U^{2 / 3} \mathrm{de}-$ notes the thickness of the film laid down at position $x$ at time $t=t_{0}(x)=R^{-1}(x)$, where $L=R(0)$ (which may be zero or positive) is the initial length of the thin films on the plates at $t=0$. Equation (23) shows that if the plate is superheated (i.e., if $\Delta T_{1}>0$ ) then liquid evaporates from the thin film which dries out locally at position $x$ at time $t=t_{0}$ $+K \bar{h}_{0}(x) / \hat{E} \Delta T_{1}$, while if the plate is subcooled (i.e., if $\Delta T_{1}$ $<0)$ then vapor condenses onto the thin film and local dry out never occurs.

For ease of presentation, we rescale $\bar{h}$ and $\hat{E}$ with $c$ and $\hat{D}$ with $c^{-1}$ in order to remove the constant $c$ from the leadingorder problem, and remove all of the overbars and hats on the variables. Furthermore, for simplicity in what follows we 
set $E=K=1$ and (unless stated otherwise) $D=1$ in all of the numerical results reported in the present work.

\section{BOTH PLATES SUPERHEATED $\left(\Delta T_{\mathrm{u}}>0\right.$ AND $\left.\Delta T_{1}=1\right)$}

If both plates are superheated (i.e., if $\Delta T_{\mathrm{u}}>0$ and $\left.\Delta T_{1}=1\right)$ then the evaporation from the capillary-statics region and any films present in the thin-film regions on both plates always causes the bubble to expand, and the dynamics of the expansion are governed by Eq. (11), which takes the form

$$
U=\frac{d R}{d t}=\frac{D E}{2 K}\left[\left(\Delta T_{\mathrm{u}}+\Delta T_{1}\right) K S+\Delta T_{\mathrm{u}} L_{\mathrm{u}}+\Delta T_{1} L_{1}\right]>0,
$$

where $L_{\mathrm{u}}=L_{\mathrm{u}}(t)$ and $L_{1}=L_{1}(t)\left(0 \leq L_{\mathrm{u}}, L_{1} \leq R\right)$ denote the total lengths of film on the upper and lower plates in $x>0$, respectively. Note that the three terms on the right hand side of Eq. (24) represent the contributions to the expansion due to the evaporation from the capillary-statics region, the evaporation from any film present in the thin-film region on the upper plate and the evaporation from any film present in the thin-film region on the lower plate, respectively.

\section{A. Delay-equation formulation for continuous films}

The liquid films laid down on the plates as the bubble expands will, in general, break up into disconnected patches of liquid as they dry. However, it is still very informative to investigate the behavior in the special case in which the films remain continuous as they dry (i.e., the special case in which there are no disconnected patches of liquid) analytically before analyzing the more general situation numerically. For continuous films we denote the position of the front of the film (where $h=h_{0}=U^{2 / 3}$ ) by $x=R\left[\tau_{\mathrm{u}}+\mathcal{T}_{\mathrm{u}}\left(\tau_{\mathrm{u}}\right)\right]=R\left[\tau_{1}+\mathcal{T}_{1}\left(\tau_{1}\right)\right]$, the position of the back of the film on the upper plate (where $h=0)$ by $x=R\left(\tau_{\mathrm{u}}\right)$, and the position of the back of the film on the lower plate (where again $h=0$ ) by $x=R\left(\tau_{1}\right)$, where $\mathcal{T}_{\mathrm{u}}=\mathcal{T}_{\mathrm{u}}\left(\tau_{\mathrm{u}}\right)$ and $\mathcal{T}_{1}=\mathcal{T}_{1}\left(\tau_{1}\right)$, given by

$$
\mathcal{T}_{\mathrm{u}}\left(\tau_{\mathrm{u}}\right)=\frac{K h_{0}}{E \Delta T_{\mathrm{u}}}=\frac{K U\left(\tau_{\mathrm{u}}\right)^{2 / 3}}{E \Delta T_{\mathrm{u}}}
$$

and

$$
\mathcal{T}_{1}\left(\tau_{1}\right)=\frac{K h_{0}}{E \Delta T_{1}}=\frac{K U\left(\tau_{1}\right)^{2 / 3}}{E \Delta T_{1}}
$$

are the lengths of time it takes for the liquid deposited on the upper and lower plates at times $t=\tau_{\mathrm{u}}$ and $t=\tau_{1}$, respectively, to dry out. Adopting this new notation, Eq. (24) can be written as

$$
\begin{aligned}
U\left[\tau_{\mathrm{u}}+\mathcal{T}_{\mathrm{u}}\left(\tau_{\mathrm{u}}\right)\right]= & \frac{D E}{2 K}\left\{\left(\Delta T_{\mathrm{u}}+\Delta T_{1}\right) K S+\Delta T_{\mathrm{u}} L_{\mathrm{u}}\left[\tau_{\mathrm{u}}+\mathcal{T}_{\mathrm{u}}\left(\tau_{\mathrm{u}}\right)\right]\right. \\
& \left.+\Delta T_{1} L_{\mathrm{l}}\left[\tau_{1}+\mathcal{T}_{\mathrm{l}}\left(\tau_{1}\right)\right]\right\}
\end{aligned}
$$

or, equivalently, as

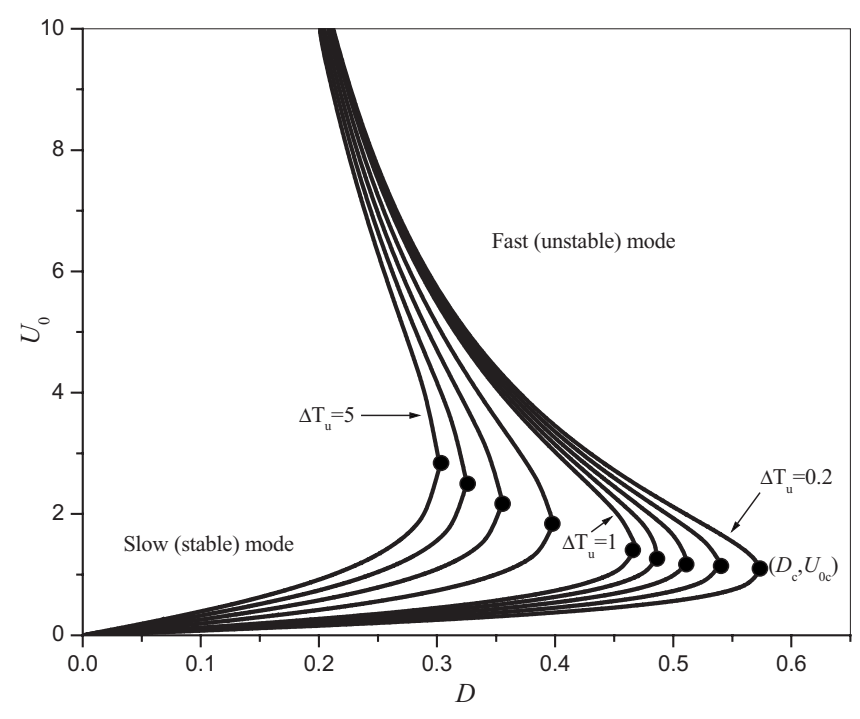

FIG. 3. The velocity of the constant-velocity continuous-film solutions $U_{0}$ plotted as a function of $D$ for $\Delta T_{\mathrm{u}}=0.2,0.4,0.6,0.8,1$, 2, 3, 4 and 5 .

$$
\begin{aligned}
U\left[\tau_{\mathrm{u}}+\mathcal{T}_{\mathrm{u}}\left(\tau_{\mathrm{u}}\right)\right]= & \frac{D E}{2 K}\left[\left(\Delta T_{\mathrm{u}}+\Delta T_{1}\right) K S+\Delta T_{\mathrm{u}} \int_{\tau_{\mathrm{u}}}^{\tau_{\mathrm{u}}+\mathcal{T}_{\mathrm{u}}\left(\tau_{\mathrm{u}}\right)} U(\hat{\tau}) d \hat{\tau}\right. \\
& \left.+\Delta T_{1} \int_{\tau_{\mathrm{l}}}^{\tau_{\mathrm{l}}+\mathcal{T}_{\mathrm{l}}\left(\tau_{\mathrm{l}}\right)} U(\hat{\tau}) d \hat{\tau}\right]
\end{aligned}
$$

Equation (28) is an integrodelay equation for $U$ with nonconstant delays $\mathcal{T}_{\mathrm{u}}$ and $\mathcal{T}_{1}$ which depend on the solution for $U$ at times $t=\tau_{\mathrm{u}}$ and $t=\tau_{1}$, respectively, according to Eqs. (25) and (26).

\section{B. Constant-velocity continuous-film solutions and their stability}

Equation (28) permits an exact continuous-film solution with constant velocity $U_{0}(>0)$ and the corresponding constant delays $\mathcal{T}_{\mathrm{u} 0}$ and $\mathcal{T}_{10}$ given by

$$
\mathcal{T}_{\mathrm{u} 0}=\frac{K U_{0}^{2 / 3}}{E \Delta T_{\mathrm{u}}} \quad \text { and } \quad \mathcal{T}_{10}=\frac{K U_{0}^{2 / 3}}{E \Delta T_{1}}
$$

Substituting $U=U_{0}, \mathcal{T}_{\mathrm{u}}=\mathcal{T}_{\mathrm{u} 0}$ and $\mathcal{T}_{1}=\mathcal{T}_{10}$ into Eq. (28) shows that $U_{0}$ satisfies

$$
\begin{aligned}
U_{0} & =\frac{D E}{2 K}\left[\left(\Delta T_{\mathrm{u}}+\Delta T_{1}\right) K S+\left(\Delta T_{\mathrm{u}} \mathcal{T}_{\mathrm{u} 0}+\Delta T_{1} \mathcal{T}_{10}\right) U_{0}\right] \\
& =\frac{D E}{2}\left(\Delta T_{\mathrm{u}}+\Delta T_{1}\right) S+D U_{0}^{5 / 3} .
\end{aligned}
$$

Figure 3 shows $U_{0}$ plotted as a function of $D$ for a range of values of $\Delta T_{\mathrm{u}}$, and shows that for $0<D<D_{\mathrm{c}}$ there are two branches of positive solutions, namely, a "fast" mode satisfying $U_{0}>U_{0 \mathrm{c}}$ and a "slow" mode satisfying $0<U_{0}<U_{0 \mathrm{c}}$, but that there are no positive solutions for $D>D_{\mathrm{c}}$, where the critical values $D_{\mathrm{c}}$ and $U_{0 \mathrm{c}}$ are given by 


$$
D_{\mathrm{c}}=\left(\frac{3}{5}\right)^{3 / 5}\left[\frac{4}{5 E\left(\Delta T_{\mathrm{u}}+\Delta T_{1}\right) S}\right]^{2 / 5}
$$

and

$$
U_{0 \mathrm{c}}=\left(\frac{3}{5 D_{\mathrm{c}}}\right)^{3 / 2}=\left[\frac{3 E\left(\Delta T_{\mathrm{u}}+\Delta T_{1}\right) S}{4}\right]^{3 / 5} .
$$

In particular, the slow mode satisfies $U_{0} \sim D E\left(\Delta T_{\mathrm{u}}\right.$ $\left.+\Delta T_{1}\right) S / 2 \rightarrow 0^{+}$and the fast mode satisfies $U_{0} \sim D^{-3 / 2} \rightarrow \infty$ in the limit $D \rightarrow 0^{+}$, while both modes satisfy $U_{0}-U_{0 \mathrm{c}}=O\left(D_{\mathrm{c}}\right.$ $-D)^{1 / 2}$ in the limit $D \rightarrow D_{\text {c }}^{-}$. For both modes the profiles of the films on both plates are linear in $x$. On the lower plate the profile has slope $E \Delta T_{1} / K U_{0}$ and increases from the value $h=0$ at the back

$$
x=R\left(t-\mathcal{T}_{10}\right)=U_{0} t-\frac{K U_{0}^{5 / 3}}{E \Delta T_{1}}
$$

to the value $h=U_{0}^{2 / 3}$ at the front $x=R(t)=U_{0} t$ according to

$$
h=U_{0}^{2 / 3}+\frac{E \Delta T_{1}}{K U_{0}}\left(x-U_{0} t\right) .
$$

The corresponding results for the profile of the film on the upper plate are obtained by replacing $\mathcal{T}_{10}$ with $\mathcal{T}_{\mathrm{u} 0}$ and $\Delta T_{1}$ with $\Delta T_{\mathrm{u}}$.

The stability of both modes can be determined by writing $U=U_{0}+U_{1}, \mathcal{T}_{\mathrm{u}}=\mathcal{T}_{\mathrm{u} 0}+\mathcal{T}_{\mathrm{u} 1}$ and $\mathcal{T}_{1}=\mathcal{T}_{10}+\mathcal{T}_{11}$ and linearizing for small $U_{1}, \mathcal{T}_{\mathrm{u} 1}$ and $\mathcal{T}_{11}$. From Eqs. (25) and (26) the first-order delays $\mathcal{T}_{\mathrm{u} 1}=\mathcal{T}_{\mathrm{u} 1}\left(\tau_{\mathrm{u}}\right)$ and $\mathcal{T}_{11}=\mathcal{T}_{11}\left(\tau_{1}\right)$ are given by

$$
\mathcal{T}_{\mathrm{u} 1}\left(\tau_{\mathrm{u}}\right)=\frac{2 K U_{1}\left(\tau_{\mathrm{u}}\right)}{3 E \Delta T_{\mathrm{u}} U_{0}^{1 / 3}}
$$

and

$$
\mathcal{T}_{11}\left(\tau_{1}\right)=\frac{2 K U_{1}\left(\tau_{1}\right)}{3 E \Delta T_{1} U_{0}^{1 / 3}},
$$

and from Eq. (28) the equation for $U_{1}$ is found to be

$$
\begin{aligned}
U_{1}\left(\tau_{\mathrm{u}}+\mathcal{T}_{\mathrm{u} 0}\right)= & \frac{D E}{2 K}\left[\left(\Delta T_{\mathrm{u}} \mathcal{T}_{\mathrm{u} 1}+\Delta T_{1} \mathcal{T}_{11}\right) U_{0}\right. \\
& \left.+\Delta T_{\mathrm{u}} \int_{\tau_{\mathrm{u}}}^{\tau_{\mathrm{u}}+\mathcal{T}_{\mathrm{u} 0}} U_{1}(\hat{\tau}) d \hat{\tau}+\Delta T_{1} \int_{\tau_{1}}^{\tau_{1}+\mathcal{T}_{10}} U_{1}(\hat{\tau}) d \hat{\tau}\right] .
\end{aligned}
$$

Equation (37) is an integrodelay equation for $U_{1}$ with (known) constant delays $\mathcal{T}_{\mathrm{u} 0}$ and $\mathcal{T}_{10}$, which has an exact solution in the form

$$
U_{1}(t) \propto \exp \left(\frac{E \beta}{K} t\right),
$$
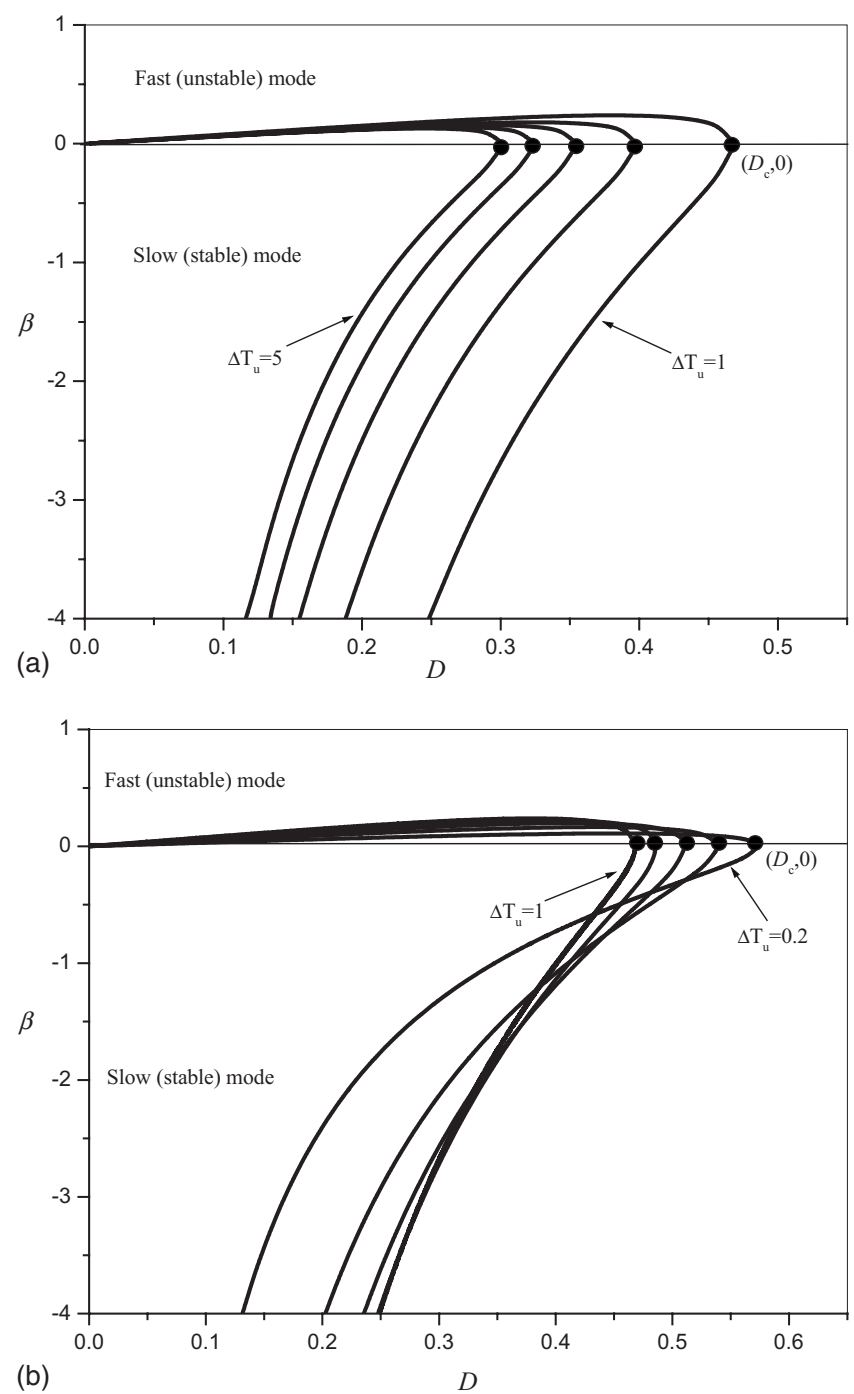

FIG. 4. The temporal growth rate of the constant-velocity continuous-film solutions $\beta$ plotted as a function of $D$ for (a) $\Delta T_{\mathrm{u}}$ $=1,2, \ldots, 5$ and (b) $\Delta T_{\mathrm{u}}=0.2,0.4, \ldots, 1$.

where the temporal growth rate $\beta=\beta\left(U_{0}, D, \Delta T_{\mathrm{u}}, \Delta T_{1}\right)$ satisfies the algebraic equation

$$
\begin{aligned}
& \frac{2 \beta}{D}\left[1-\frac{D U_{0}^{2 / 3}}{3}\left\{\exp \left(-\frac{\beta U_{0}^{2 / 3}}{\Delta T_{\mathrm{u}}}\right)+\exp \left(-\frac{\beta U_{0}^{2 / 3}}{\Delta T_{1}}\right)\right\}\right] \\
& \quad=\Delta T_{\mathrm{u}}\left[1-\exp \left(-\frac{\beta U_{0}^{2 / 3}}{\Delta T_{\mathrm{u}}}\right)\right]+\Delta T_{1}\left[1-\exp \left(-\frac{\beta U_{0}^{2 / 3}}{\Delta T_{1}}\right)\right] .
\end{aligned}
$$

Figure 4 shows $\beta$ plotted as a function of $D$ for a range of values of $\Delta T_{\mathrm{u}}$, and shows that $\beta>0$ for the fast mode and $\beta<0$ for the slow mode. In particular, for the fast mode $\beta \sim \hat{\beta} D \rightarrow 0^{+}$in the limit $D \rightarrow 0^{+}$, where $\hat{\beta}=\hat{\beta}\left(\Delta T_{\mathrm{u}}, \Delta T_{1}\right)$ satisfies the algebraic equation 


$$
\begin{aligned}
2 \hat{\beta}[1 & \left.-\frac{1}{3}\left\{\exp \left(-\frac{\hat{\beta}}{\Delta T_{\mathrm{u}}}\right)+\exp \left(-\frac{\hat{\beta}}{\Delta T_{\mathrm{l}}}\right)\right\}\right] \\
& =\Delta T_{\mathrm{u}}\left[1-\exp \left(-\frac{\hat{\beta}}{\Delta T_{\mathrm{u}}}\right)\right]+\Delta T_{1}\left[1-\exp \left(-\frac{\hat{\beta}}{\Delta T_{1}}\right)\right],
\end{aligned}
$$

and for the slow mode

$$
\beta \sim \frac{\max \left(\Delta T_{\mathrm{u}}, \Delta T_{1}\right)}{U_{0}^{2 / 3}} \log \left(\frac{D U_{0}^{2 / 3}}{3}\right)=O\left(D^{-2 / 3} \log D\right) \rightarrow-\infty
$$

in the limit $D \rightarrow 0^{+} \quad[17]$, while for both modes $\beta=O\left(D_{\mathrm{c}}-D\right)^{1 / 2} \rightarrow 0$ in the limit $D \rightarrow D_{\mathrm{c}}^{-}$. Thus the fast mode with $U_{0}>U_{0 \mathrm{c}}$ is always unstable, while the slow mode with $0<U_{0}<U_{0 \mathrm{c}}$ is always stable. These results are qualitatively different from the corresponding results obtained by WDB who found a single unstable mode with velocity $U_{0}=D^{-3 / 2}$ (corresponding to the limit $D \rightarrow 0^{+}$of the present fast mode) for all values of $D$ [18].

\section{No initial films}

If there are no films on the plates initially [i.e., if $L=R(0)=0]$ then the initial expansion of the bubble is driven solely by the evaporation from the capillary-statics region. Until the film laid down on the hotter plate starts to dry out at $x=0$ at $t=K \mathcal{U}_{0}^{2 / 3} / E \max \left(\Delta T_{\mathrm{u}}, \Delta T_{1}\right)$, where $\mathcal{U}_{0}=U(0)$ is the initial velocity of the bubble at $t=0$, we have $L_{\mathrm{u}}=L_{\mathrm{l}}=R$, and so from Eq. (24)

$$
R=K S\left[\exp \left(\frac{D E\left(\Delta T_{\mathrm{u}}+\Delta T_{1}\right)}{2 K} t\right)-1\right] .
$$

In particular, the initial expansion of the bubble is given by $U=\mathcal{U}_{0}+\mathcal{U}_{1} t+O\left(t^{2}\right)$, where $\mathcal{U}_{0}(>0)$ and $\mathcal{U}_{1}(>0)$ are given

$$
\mathcal{U}_{0}=\frac{D E\left(\Delta T_{\mathrm{u}}+\Delta T_{1}\right) S}{2}
$$

and

$$
\mathcal{U}_{1}=\left(\frac{D E\left(\Delta T_{\mathrm{u}}+\Delta T_{1}\right)}{2}\right)^{2} \frac{S}{K} .
$$

Solving Eq. (24) numerically for a range of values of $D$ reveals that the eventual behavior of the bubble is qualitatively different for $0<D<D_{\mathrm{c}}$ and $D>D_{\mathrm{c}}$, where the critical value $D_{\mathrm{c}}$ is given by Eq. (31). Specifically, we find that for $0<D<D_{\text {c }}$ the bubble eventually expands with the constant velocity of the stable slow mode calculated in Sec. IV B above but that for $D>D_{\text {c }}$ no constant-velocity solutions exists and the bubble eventually expands exponentially according to

$$
R=O\left(\exp \left[\frac{D E\left(\Delta T_{\mathrm{u}}+\Delta T_{1}\right)}{2 K} t\right]\right) \rightarrow \infty
$$

as $t \rightarrow \infty$. These results are illustrated in Fig. 5 which shows two examples of the numerically calculated evolution of the
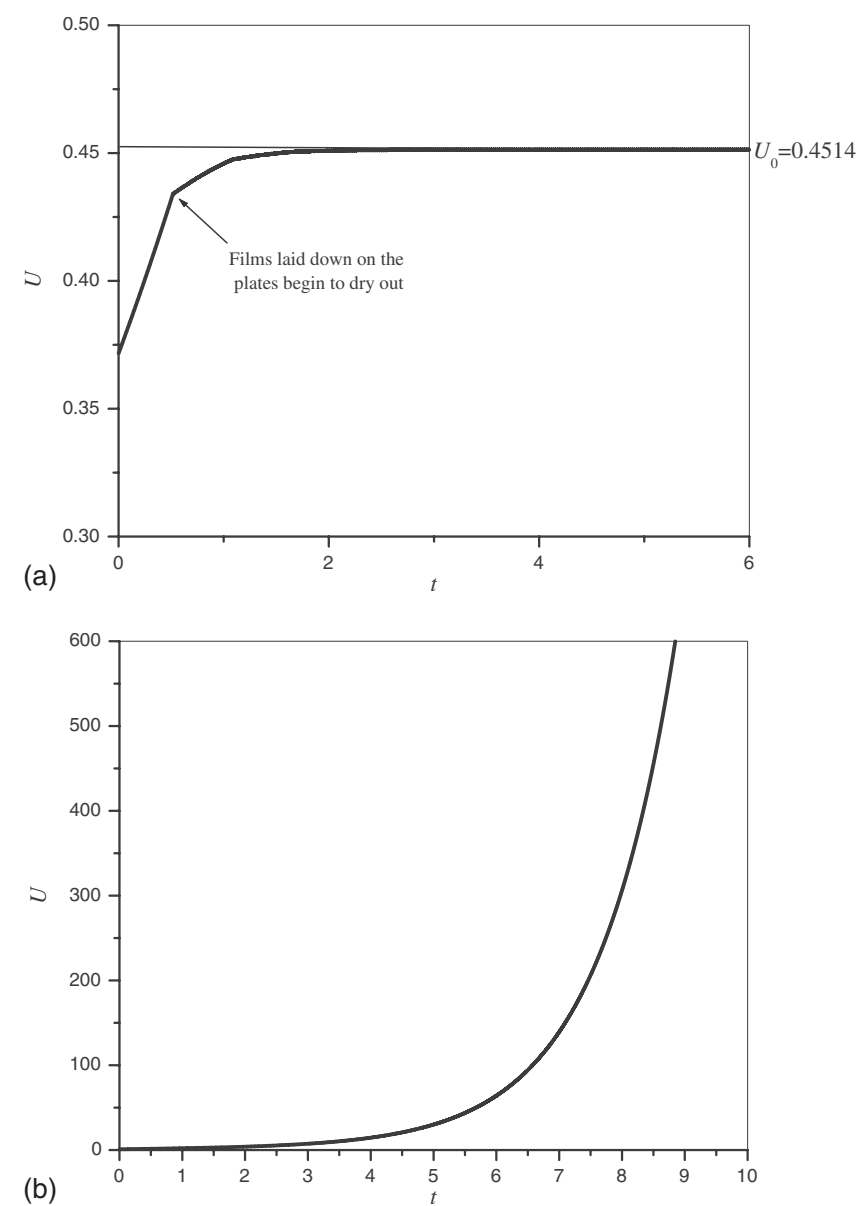

FIG. 5. The velocity of the bubble $U$ plotted as a function of time $t$ in the cases (a) $D=0.3 \quad\left(<D_{\text {c }} \simeq 0.4682\right)$ and (b) $D=0.8$ $\left(>D_{c}\right)$ in the absence of any initial films when both plates are superheated equally, i.e., when $\Delta T_{\mathrm{u}}=\Delta T_{1}=1$.

bubble in the case when both plates are superheated equally, i.e., when $\Delta T_{\mathrm{u}}=\Delta T_{1}=1$. Specifically, Fig. 5(a) illustrates that in the case $D=0.3\left(<D_{\mathrm{c}} \simeq 0.4682\right)$ the bubble velocity approaches the limiting value of $U_{0} \simeq 0.4514\left(<U_{0 \mathrm{c}} \simeq 1.4560\right)$ in the limit $t \rightarrow \infty$, while Fig. 5(b) illustrates that in the case $D=0.8\left(>D_{\text {c }}\right)$ the bubble eventually expands exponentially according to Eq. (45). Note that the "corners" in $U$ discernible in Fig. 5(a) are not the result of numerical or plotting inaccuracies, but are caused by the local dry out of the films on the plates, which results in a discontinuous change in the local mass flux and hence a discontinuous change in the slope of $U$ as a function of $t$. For example, the corner highlighted in Fig. 5(a) corresponds to the instant $t \simeq 0.5170$ at which the films of thickness $\mathcal{U}_{0}^{2 / 3} \simeq 0.5170$ laid down at $x=0$ on both plates at $t=0$ dry out creating dry patches at $x=0$ on both plates. Since the thickness of the films laid down on the plates, and hence their subsequent contribution to the total mass flux, depends on the current value of $U$, "echoes" of this event occur in the subsequent evolution of the bubble.

\section{Uniform initial films}

If there are uniform films of length $L$ and thickness $H$ on both plates initially [i.e., if $R(0)=L$ and $h_{0}=H$ for $0 \leq x \leq L$ ] 


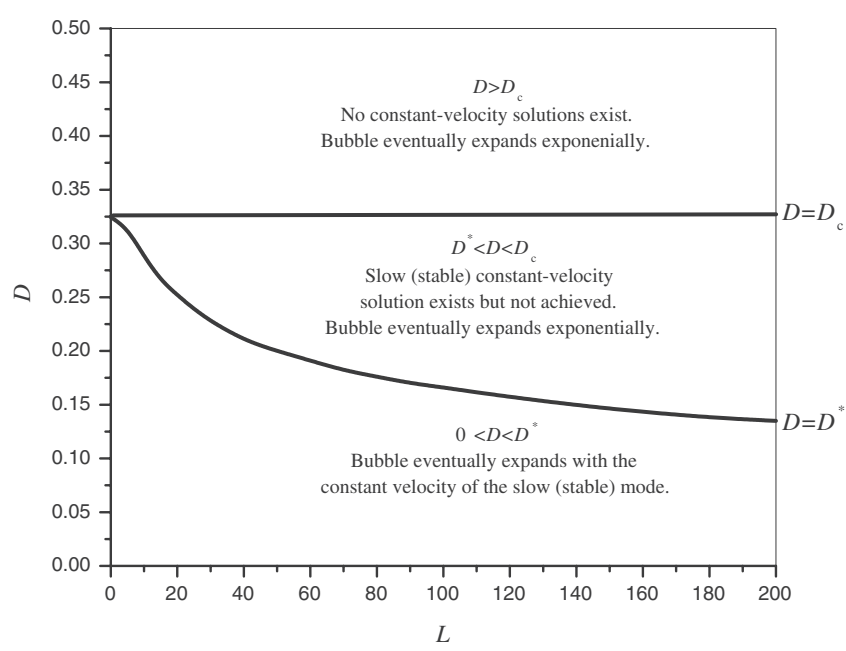

FIG. 6. A plot of the $(L, D)$ parameter plane showing how the numerically calculated curve $D=D^{*}$ divides the region in which the bubble eventually expands with the constant velocity of the slow mode from the region in which it eventually expands exponentially.

then the initial expansion of the bubble is driven by the evaporation from both the capillary-statics region and the initial films. For simplicity in what follows we set $H=1$ in all of the numerical results reported in the present work. When the initial films on the upper and lower plates dry out at

$$
t=t_{\mathrm{cu}}=\frac{K H}{E \Delta T_{\mathrm{u}}} \quad \text { and } \quad t=t_{\mathrm{cl}}=\frac{K H}{E \Delta T_{1}},
$$

this causes discontinuous drops in $U$ of magnitude $D E L \Delta T_{\mathrm{u}} / 2 K$ and $D E L \Delta T_{1} / 2 K$ at those instants, respectively, and this may occur either before or after the film laid down on the hotter plate starts to dry out at $x=L$ at $t=K \mathcal{U}_{0}^{2 / 3} / E \max \left(\Delta T_{\mathrm{u}}, \Delta T_{1}\right)$. Until the first dry out occurs we again have $L_{\mathrm{u}}=L_{\mathrm{l}}=R$ and hence

$$
R=(K S+L) \exp \left(\frac{D E\left(\Delta T_{\mathrm{u}}+\Delta T_{1}\right)}{2 K} t\right)-K S .
$$

In particular, the initial expansion is given by $U=\mathcal{U}_{0}+\mathcal{U}_{1} t$ $+O\left(t^{2}\right)$, where $\mathcal{U}_{0}(>0)$ and $\mathcal{U}_{1}(>0)$ are given

$$
\mathcal{U}_{0}=\frac{D E\left(\Delta T_{\mathrm{u}}+\Delta T_{1}\right)}{2 K}(K S+L)
$$

and

$$
\mathcal{U}_{1}=\left(\frac{D E\left(\Delta T_{\mathrm{u}}+\Delta T_{1}\right)}{2 K}\right)^{2}(K S+L) .
$$

Note that in the special case $L=0$ Eqs. (47)-(49) reduce to the corresponding expressions in the case of no initial films given by Eqs. (42)-(44) in Sec. IV D above. Solving Eq. (24) numerically for a range of values of $D$ reveals that the eventual behavior of the bubble is again qualitatively different for different values of $D$, but that the presence of the initial films makes the situation a little more complicated than when they are absent. Specifically, we find that while a stable constantvelocity solution is again possible for all values of $D$ in the range $0<D<D_{\text {c }}$, it is eventually attained only when $D$ is
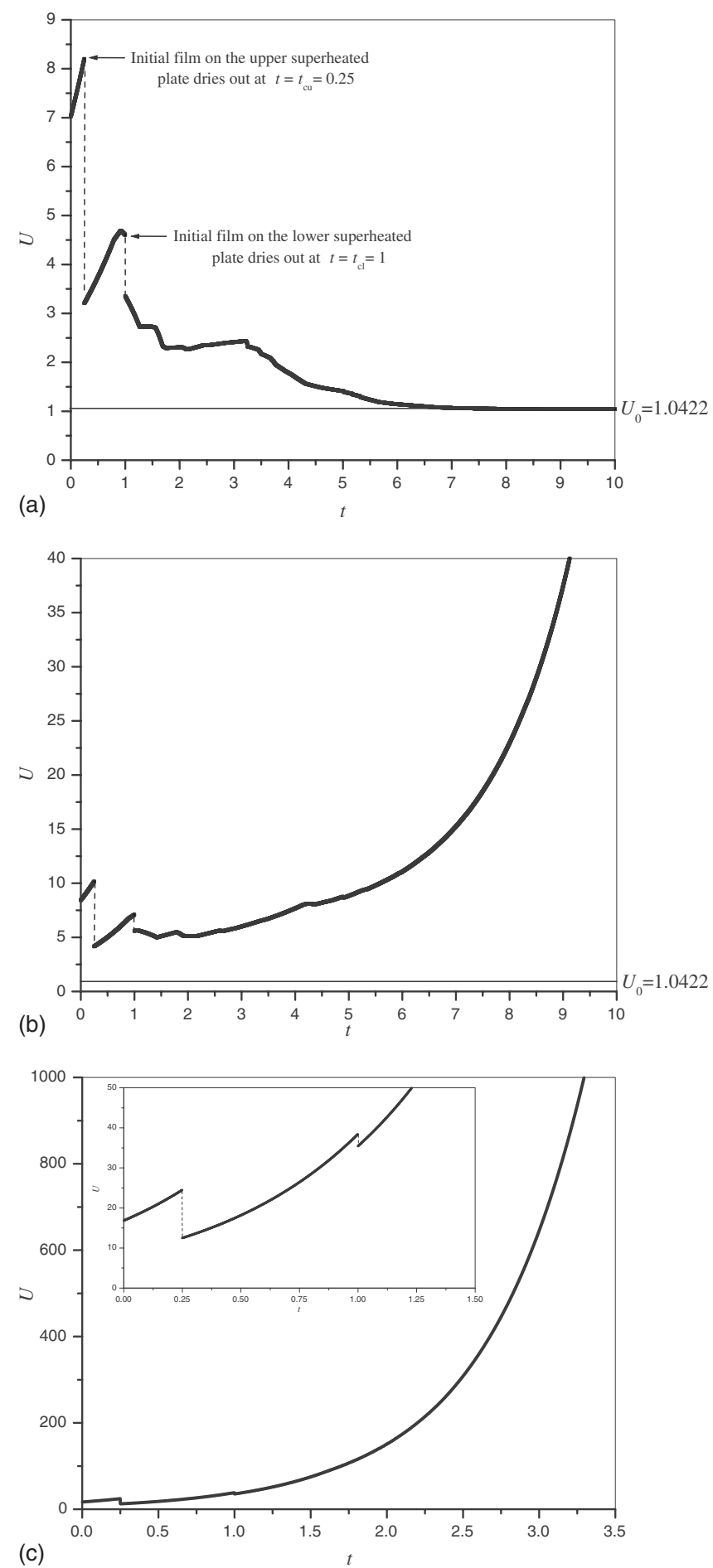

FIG. 7. The velocity of the bubble $U$ plotted as a function of time $t$ in the cases (a) $D=0.25\left(<D^{*} \simeq 0.2890\right)$, (b) $D=0.3\left(D^{*}\right.$ $\left.<D<D_{\mathrm{c}} \simeq 0.3245\right)$ and (c) $D=0.6\left(>D_{\mathrm{c}}\right)$ with initial films of length $L=10$ when both plates are superheated unequally with $\Delta T_{\mathrm{u}}=4$ and $\Delta T_{1}=1$. In part (c) the insert shows an enlarged version of the plot for $0 \leq t \leq 1.5$. Note the very different vertical scales used in parts (a), (b) and (c).

less than a critical value denoted by $D^{*}\left(<D_{\text {c }}\right)$. Thus for 0 $<D<D^{*}$ the bubble eventually expands with the constant velocity of the stable slow mode calculated in Sec. IV B above, while for $D>D^{*}$ we find that the bubble eventually 


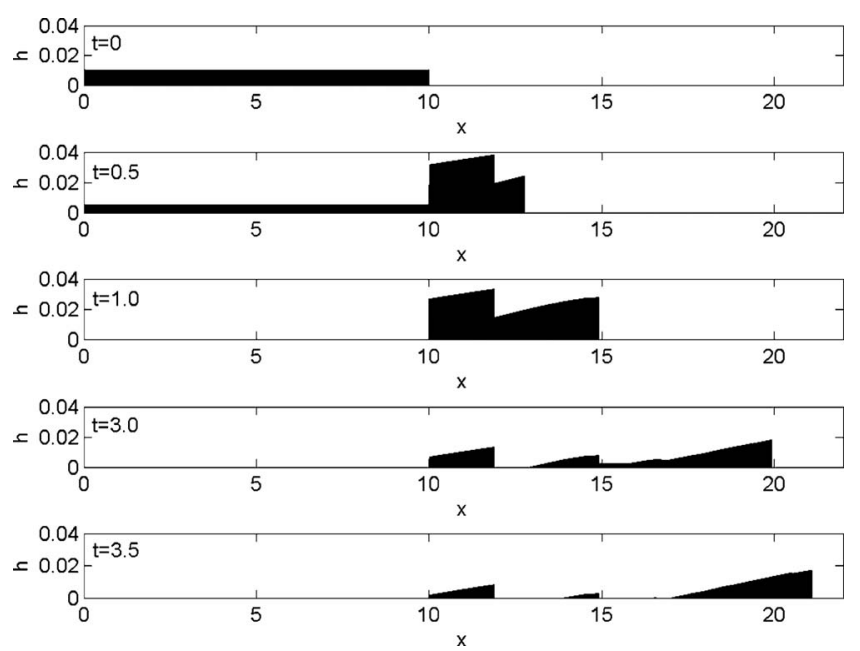

FIG. 8. The profile of the film on the lower plate in the case $D=0.25$ with initial films of length $L=10$ when both plates are superheated unequally with $\Delta T_{\mathrm{u}}=4$ and $\Delta T_{1}=1$ at $t=0,0.5,1,3$ and 3.5 .

expands exponentially according to Eq. (45). In general, the value of $D^{*}$ has to be calculated numerically. Figure 6 shows $D^{*}$ plotted as a function of $L$, and, in particular, shows that $D^{*} \rightarrow D_{\text {c }}^{-}$as $L \rightarrow 0^{+}$. Figure 6 also shows how the curve $D$ $=D^{*}$ divides the $(L, D)$ parameter plane into the region in which the bubble eventually expands with the constant velocity of the slow mode from the region in which it eventually expands exponentially. These results are illustrated in Fig. 7 which shows three examples of the numerically calculated evolution of the bubble in the case $L=10$ when $\Delta T_{\mathrm{u}}=4$ and $\Delta T_{1}=1$. Specifically, Fig. 7 shows the evolution in the cases (a) $D=0.25 \quad\left(<D^{*} \simeq 0.2890\right)$, (b) $D=0.3$ $\left(D^{*}<D<D_{\mathrm{c}} \simeq 0.3245\right)$ and (c) $D=0.6\left(>D_{\mathrm{c}}\right)$. In particular, Fig. 7(b) shows the evolution in a case in which a stable constant-velocity solution exists but is not achieved. In addition to the corners in $U$ arising from the local dry out of the films like those seen previously in Fig. 5(a), Fig. 7 also clearly shows the discontinuous drops in $U$ arising from the dry out of the initial films on the upper and lower plates at $t_{\mathrm{cu}}=0.25$ and $t_{\mathrm{cl}}=1$, respectively.

Figure 8 shows the profile of the film on the lower plate in the case $D=0.25$ at various times. In particular, Fig. 8 shows the disappearance of the uniform initial film of length $L=10$ at time $t=t_{\mathrm{cl}}=1$ and the presence of one disconnected patch of liquid at $t=3$ and two disconnected patches of liquid at $t=3.5$ created as the nonuniform film which is laid down on the plate as the bubble expands dries out locally.

\section{BOTH PLATES SUBCOOLED $\left(\Delta T_{\mathrm{u}}<0\right.$ AND $\left.\Delta T_{1}=-1\right)$}

If both plates are subcooled (i.e., if $\Delta T_{\mathrm{u}}<0$ and $\left.\Delta T_{1}=-1\right)$ then the condensation onto the capillary-statics region and the thin-film regions on both plates always causes the bubble to contract, and the dynamics of the contraction are governed by Eq. (11), which takes the simple form

$$
U=\frac{d R}{d t}=\frac{D E\left(\Delta T_{\mathrm{u}}+\Delta T_{1}\right)}{2 K}(K S+R)<0 .
$$

Note that the two terms on the right hand side of Eq. (50) represent the contributions to the contraction due to the con-

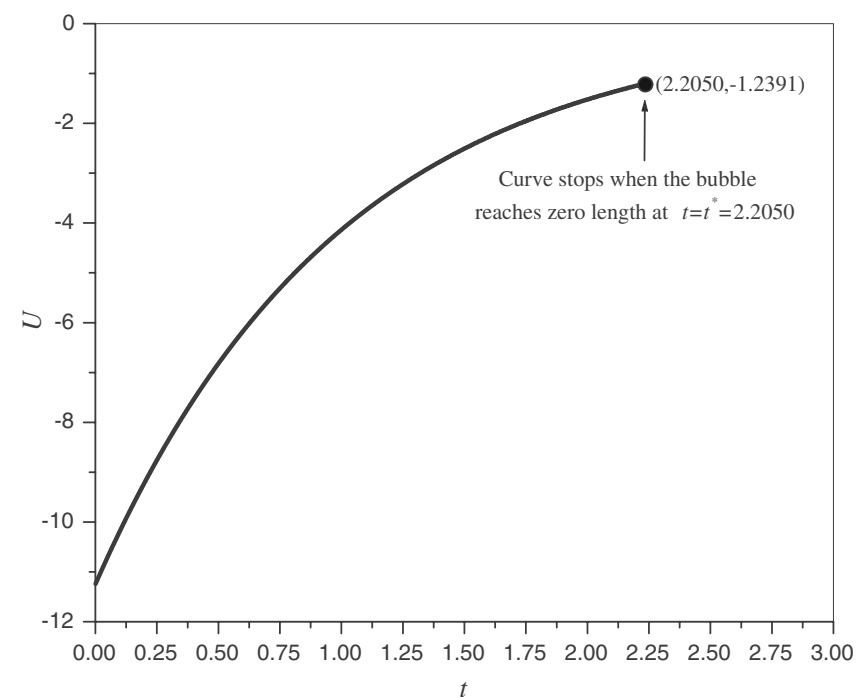

FIG. 9. The velocity of the bubble $U$ plotted as a function of time $t$ for initial films of length $L=10$ when both plates are subcooled equally, i.e., when $\Delta T_{\mathrm{u}}=\Delta T_{1}=-1$. Note that the curve stops when the bubble reaches zero length at $t=t^{*}=2.2050$.

densation onto the capillary-statics region and the condensation onto the thin-film regions on the upper and lower plates, respectively.

If there are no initial films [i.e., if $L=R(0)=0$ ] then $R$ immediately becomes negative (which is, of course, physically impossible), i.e., the model fails immediately. However, if there are uniform initial films of length $L$ (i.e., if $R(0)=L$ ) then the bubble collapses exponentially according to Eq. (47). In particular, the initial contraction of the bubble is given by $U=\mathcal{U}_{0}+\mathcal{U}_{1} t+O\left(t^{2}\right)$, where $\mathcal{U}_{0}(<0)$ and $\mathcal{U}_{1}(>0)$ are given by Eqs. (48) and (49). Eventually, the model fails when the bubble reaches zero length at the finite time $t=t^{*}$ given by

$$
t^{*}=-\frac{2 K}{D E\left(\Delta T_{\mathrm{u}}+\Delta T_{1}\right)} \log \left(1+\frac{L}{K S}\right) .
$$

Figure 9 shows the evolution of the bubble in the case $L=10$ when both plates are subcooled equally, i.e., when $\Delta T_{\mathrm{u}}=\Delta T_{1}=-1$, in which case the model fails when the bubble reaches zero length at $t=t^{*}=2.2050$.

\section{UPPER PLATE SUBCOOLED AND LOWER PLATE SUPERHEATED $\left(\Delta T_{\mathrm{u}}<0\right.$ AND $\left.\Delta T_{1}=1\right)$}

If the upper plate is subcooled and the lower plate is superheated (i.e., if $\Delta T_{\mathrm{u}}<0$ and $\Delta T_{1}=1$ ) then the net effect of the evaporation from and condensation onto the capillarystatics region, the condensation onto the thin-film region on the upper plate and the evaporation from any film present in the thin-film region on the lower plate may cause the bubble to expand or contract, and the dynamics are governed by Eq. (11), which takes the form 


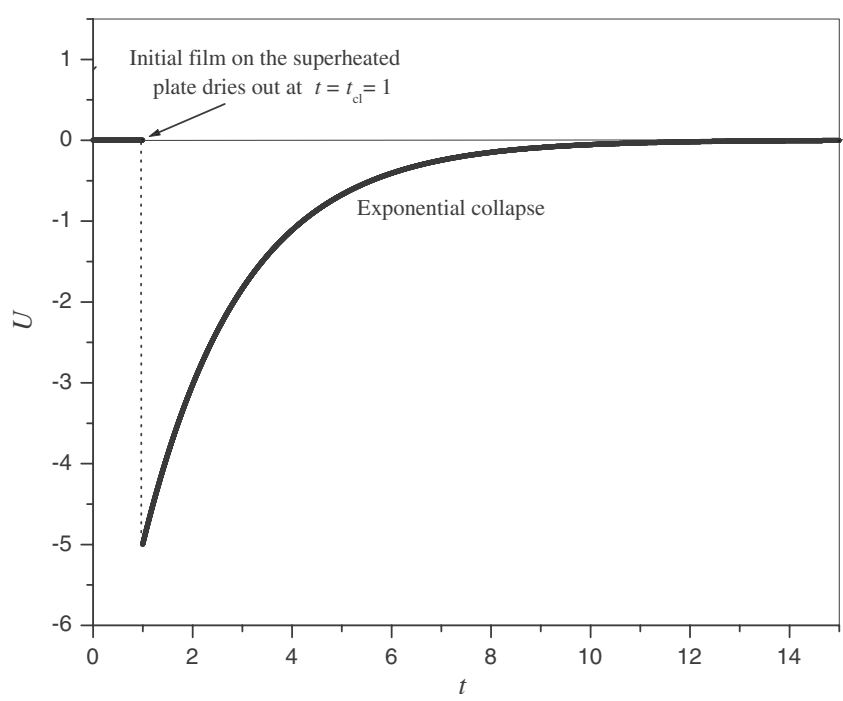

FIG. 10. The velocity of the bubble $U$ plotted as a function of time $t$ for initial films of length $L=10$ when the upper plate is subcooled and the lower plate is superheated with equal magnitude, i.e., when $-\Delta T_{\mathrm{u}}=\Delta T_{1}=1$.

$$
U=\frac{d R}{d t}=\frac{D E}{2 K}\left[\left(\Delta T_{\mathrm{u}}+\Delta T_{1}\right) K S+\Delta T_{\mathrm{u}} R+\Delta T_{1} L_{1}\right],
$$

where $L_{1}=L_{1}(t)\left(0 \leq L_{1} \leq R\right)$ is again the total length of film on the lower plate in $x>0$. Note that the three terms on the right hand side of Eq. (52) represent the contributions to the expansion or contraction due to the evaporation from and condensation onto the capillary-statics region, the condensation onto the thin-film region on the subcooled upper plate and the evaporation from any film present in the thin-film region on the superheated lower plate, respectively.

\section{A. Magnitude of subcooling and superheating equal $\left(-\Delta T_{\mathrm{u}}=\Delta T_{1}=1\right)$}

If the magnitude of the subcooling is equal to that of the superheating (i.e., the special case $-\Delta T_{\mathrm{u}}=\Delta T_{1}=1$ ) then the behavior of the bubble depends on whether or not any initial films are present. If there are no initial films then the evaporation from the lower half of the semicircular cap is exactly balanced by the condensation onto the upper half of the cap, and hence $U \equiv 0$, i.e., the bubble remains stationary for all time. However, if there are uniform initial films of length $L$ then the bubble remains stationary until the initial film on the superheated plate dries out at $t=t_{\mathrm{cl}}$ given by Eq. (46), and thereafter for $t>t_{\mathrm{cl}}$ the bubble collapses exponentially to zero length in an infinite time according to

$$
R=L \exp \left(-\frac{D E\left(-\Delta T_{\mathrm{u}}\right)}{2 K}\left(t-t_{\mathrm{cl}}\right)\right)
$$

Figure 10 shows the evolution of the bubble in the case $L=10$. In particular, Fig. 10 shows both the discontinuous drop in $U$ of magnitude $D E L \Delta T_{1} / 2 K=5$ at $t=t_{\mathrm{cl}}=1$ when the initial film on the superheated plate dries out, and the subsequent exponential decay of $U$.

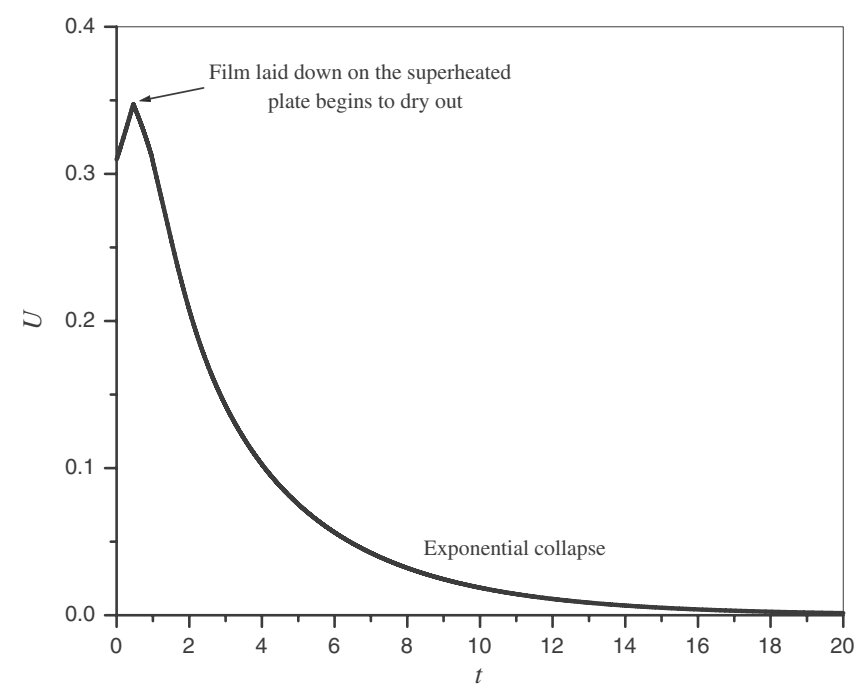

FIG. 11. The velocity of the bubble $U$ plotted as a function of time $t$ in the absence of any initial films when $\Delta T_{\mathrm{u}}=-0.5$ and $\Delta T_{1}=1$.

\section{B. Magnitude of subcooling less than that of superheating $\left(-\Delta T_{\mathrm{u}}<\Delta T_{1}=1\right)$}

If the magnitude of the subcooling is less than that of the superheating (i.e., if $-\Delta T_{\mathrm{u}}<\Delta T_{1}=1$ ) then the bubble always expands initially, but its ultimate fate depends on whether or not the magnitude of the subcooling $-\Delta T_{\mathrm{u}}(>0)$ is greater or less than a critical value, denoted by $-\Delta T_{\mathrm{uc}}(>0)$. Specifically, if $\left|\Delta T_{\mathrm{uc}}\right|<\left|\Delta T_{\mathrm{u}}\right|<\Delta T_{1}=1$ then once all of the film on the superheated plate has eventually disappeared (i.e., when $\left.L_{1}=0\right)$ the bubble collapses exponentially to the finite length $R=R_{\infty}>0$ given by

$$
R_{\infty}=\frac{K S\left(\Delta T_{\mathrm{u}}+\Delta T_{1}\right)}{\left(-\Delta T_{\mathrm{u}}\right)}
$$

according to

$$
R-R_{\infty}=O\left(\exp \left[-\frac{D E\left(-\Delta T_{\mathrm{u}}\right)}{2 K} t\right]\right)
$$

as $t \rightarrow \infty$. On the other hand if $\left|\Delta T_{\mathrm{u}}\right|<\left|\Delta T_{\mathrm{uc}}\right|$ then the bubble always expands and eventually does so exponentially according to Eq. (45) as $t \rightarrow \infty$. Note that until the film laid down on the superheated plate starts to dry out at $x=0$ at $t$ $=K \mathcal{U}_{0}^{2 / 3} / E \Delta T_{1}$, the evolution of the bubble is again described by Eqs. (42)-(44) when there are no initial films, and by Eqs. (47)-(49) when there are uniform initial films.

If there are no initial films the value of $-\Delta T_{\mathrm{uc}}$ is found numerically to be 0.0097 . Figure 11 shows the numerically calculated evolution of the bubble in the case $\Delta T_{\mathrm{u}}=-0.5$, which satisfies $\left|\Delta T_{\mathrm{uc}}\right|<\left|\Delta T_{\mathrm{u}}\right|<\Delta T_{1}=1$.

If there are uniform initial films the value of $-\Delta T_{\mathrm{uc}}$ depends on the values of $L$ and $H$. Figure 12 shows how the numerically calculated curve $-\Delta T_{\mathrm{u}}=-\Delta T_{\mathrm{uc}}$ divides the $\left(L,-\Delta T_{\mathrm{u}}\right)$ parameter plane into the region in which the bubble eventually expands exponentially from the region in which it eventually collapses to the finite length $R_{\infty}$. In particular, Fig. 12 shows that $-\Delta T_{\text {uc }}$ increases monotonically 


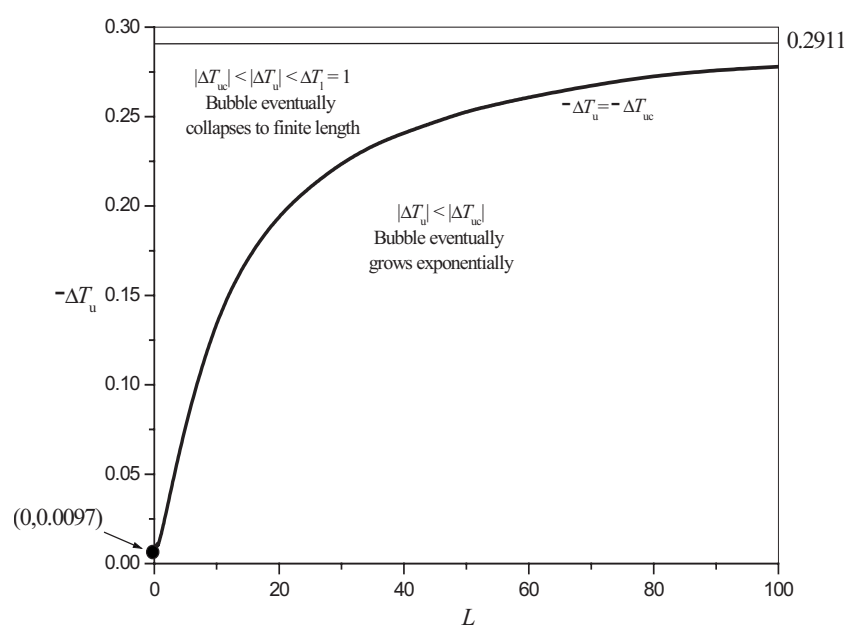

FIG. 12. A plot of the $\left(L,-\Delta T_{\mathrm{u}}\right)$ parameter plane showing how the numerically calculated curve $-\Delta T_{\mathrm{u}}=-\Delta T_{\mathrm{uc}}$ divides the region in which the bubble eventually expands exponentially from the region in which it eventually collapses to the finite length $R_{\infty}$.

from 0.0097 when $L=0$ towards 0.2911 in the limit $L \rightarrow \infty$. Figure 13 shows the numerically calculated evolution of the bubble in the case $L=50$ when $\Delta T_{\mathrm{u}}=-0.26$ and $\Delta T_{\mathrm{u}}=-0.31$, both of which satisfy $\left|\Delta T_{\mathrm{uc}}\right|<\left|\Delta T_{\mathrm{u}}\right|<\Delta T_{1}=1$, where $-\Delta T_{\mathrm{uc}}$ $\simeq 0.2527$. In particular, Fig. 13 shows that the dynamics can be considerably more complicated than those in the case of no initial film. Specifically, Fig. 13(b) shows that an unexpected "waiting time" behavior in which the bubble remains almost stationary for a finite period of time can occur.

\section{Magnitude of subcooling greater than that of superheating

$$
\left(-\Delta T_{\mathrm{u}}>\Delta T_{1}=1\right)
$$

If the magnitude of the subcooling is greater than that of the superheating (i.e., if $-\Delta T_{\mathrm{u}}>\Delta T_{1}=1$ ) then the bubble always contracts.

If there are no initial films then the model fails immediately as in Sec. V above. However, if there are uniform initial films of length $L$ then $L_{1}=R$ until all the film on the superheated plate dries out at $t=t_{\mathrm{cl}}$ given by Eq. (46), and so for $0 \leq t<t_{\mathrm{cl}}$ the bubble contracts exponentially according to Eq. (47), reaching the length $R=R_{\mathrm{c}}=R\left(t_{\mathrm{cl}}\right)$ at $t=t_{\mathrm{cl}}$. Thereafter for $t>t_{\mathrm{cl}}$ the bubble collapses exponentially according to

$$
R=R_{\infty}+\left(R_{\mathrm{c}}-R_{\infty}\right) \exp \left[-\frac{D E\left(-\Delta T_{\mathrm{u}}\right)}{2 K}\left(t-t_{\mathrm{cl}}\right)\right],
$$

where $R_{\infty}<0$ (which is now negative) is again given by Eq. (54), until eventually the model fails when the bubble reaches zero length at the finite time $t=t^{*}$ given by

$$
t^{*}=t_{\mathrm{cl}}+\frac{2 K}{D E\left(-\Delta T_{\mathrm{u}}\right)} \log \left(1+\frac{R_{\mathrm{c}}}{\left(-R_{\infty}\right)}\right) .
$$

\section{PHYSICAL VALUES}

In order to illustrate typical physical situations in which the analysis of WDB and the present analysis apply, it is
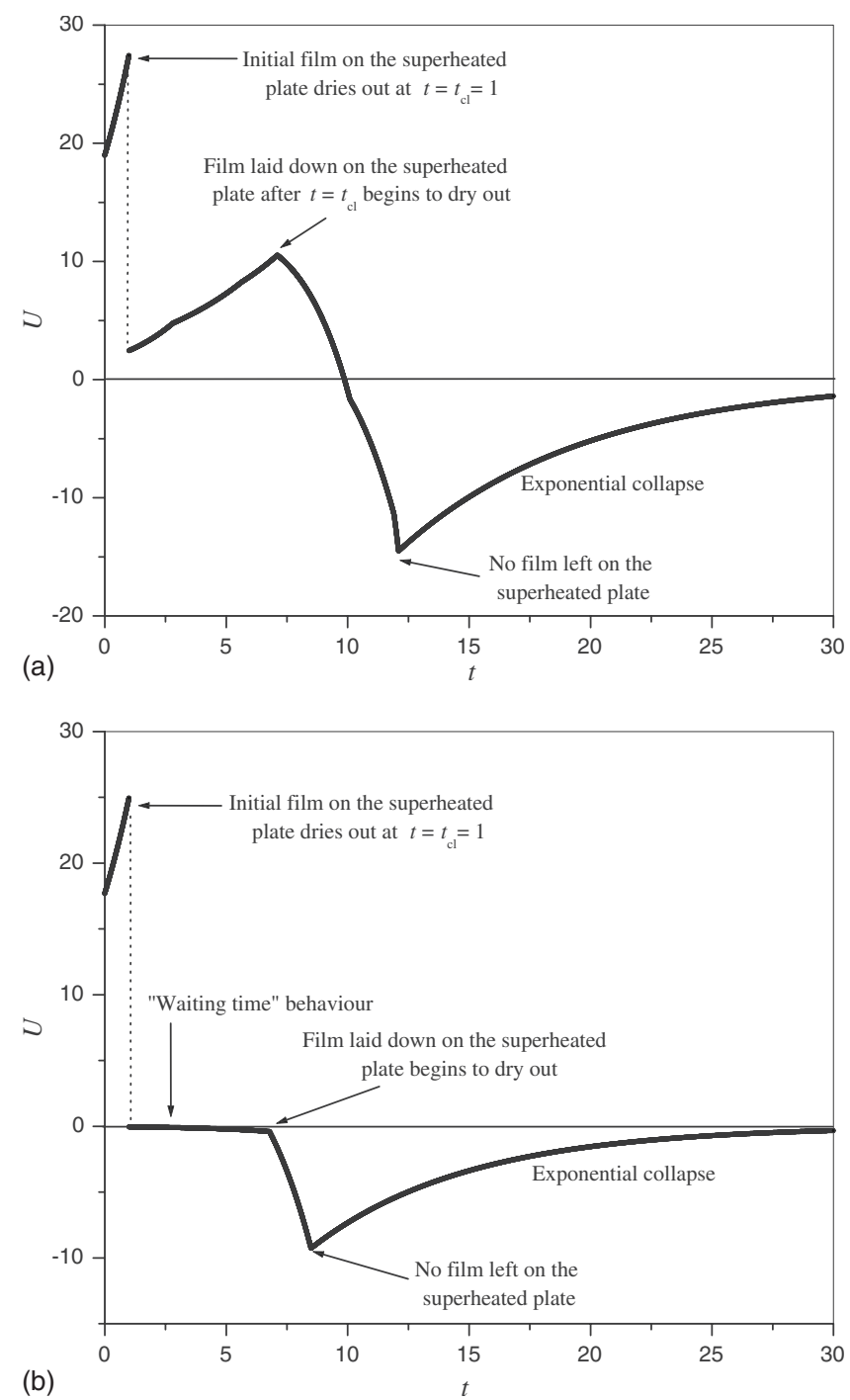

FIG. 13. The velocity of the bubble $U$ plotted as a function of time $t$ in the cases (a) $\Delta T_{\mathrm{u}}=-0.26$ and (b) $\Delta T_{\mathrm{u}}=-0.31$ with initial films of length $L=50$ when $\Delta T_{1}=1$.

informative to consider the typical physical parameter values for water and mercury listed in Table I and the corresponding values of the nondimensional parameters $P, C, D, E$, and $K$ listed in Table II. Both analyses are based on the assumption that the capillary number is small, $C \ll 1$, and hence that $d \gg O\left(10^{-9}\right) \mathrm{m}$ for water and $d \gg O\left(10^{-10}\right) \mathrm{m}$ for mercury, and that the density ratio is large, $D=O\left(C^{-2 / 3}\right) \gg 1$. The analysis of WDB applies when $P=O(1), K=O\left(C^{2 / 3}\right) \ll 1$ and $E=O\left(C^{4 / 3}\right) \ll 1$ and so corresponds to physical situations with "large" gaps and "small" superheating or subcooling whereas the present analysis applies when $P=o(1)$, $K=O(1)$ and $E=O\left(C^{2 / 3}\right) \ll 1$ and so corresponds to physical situations with "small" gaps and "large" superheating or subcooling. Thus (assuming for definiteness that the accommodation coefficient $\alpha=1$ [19]) while the analysis of WDB is applicable to water when $d=O\left(10^{-4}\right) \mathrm{m}$ and $\left|\Delta T_{1}\right|$ $=O\left(10^{-4}\right) \mathrm{K}$, the present analysis is applicable to mercury when $d=O\left(10^{-6}\right) \mathrm{m}$ and $\left|\Delta T_{1}\right|=O\left(10^{-2}\right) \mathrm{K}$. In particular, setting $K=1$ and $E=C^{2 / 3}$ for mercury corresponds to choosing $d=6 \times 10^{-6} \mathrm{~m}$ and $\left|\Delta T_{1}\right|=0.03 \mathrm{~K}$, and the present theo- 
TABLE I. Typical physical parameter values for water and mercury.

\begin{tabular}{lcccc}
\hline \hline Physical quantity & Symbol & Value for water & Value for mercury & Units \\
\hline Saturation temperature & $T_{\mathrm{S}}$ & 373 & 630 & $\mathrm{~K}$ \\
Density & $\rho$ & 960 & $1.3 \times 10^{4}$ & $\mathrm{~kg} \mathrm{~m}^{-3}$ \\
Density of vapor & $\rho^{(V)}$ & 0.6 & 4 & $\mathrm{~kg} \mathrm{~m}^{-3}$ \\
Viscosity & $\nu$ & $3 \times 10^{-7}$ & $7 \times 10^{-8}$ & $\mathrm{~m}^{2} \mathrm{~s}^{-1}$ \\
Thermal conductivity & $k$ & 0.68 & 8.3 & $\mathrm{Wm}^{-1} \mathrm{~K}^{-1}$ \\
Thermal diffusivity & $\kappa$ & $1.7 \times 10^{-7}$ & $4 \times 10^{-6}$ & $\mathrm{~m}^{2} \mathrm{~s}^{-1}$ \\
Latent heat of vaporization & $\mathcal{L}$ & $2.3 \times 10^{6}$ & $3 \times 10^{5}$ & $\mathrm{~J} \mathrm{~kg}^{-1}$ \\
Surface tension & $\sigma$ & 0.06 & 0.5 & $\mathrm{~N} \mathrm{~m}^{-1}$ \\
Molecular weight & $M_{\mathrm{W}}$ & 0.018 & 0.2 & $\mathrm{~kg} \mathrm{~mol}^{-1}$ \\
\hline \hline
\end{tabular}

retical prediction for the (dimensional) initial expansion or contraction velocity of a bubble between equally subcooled or superheated plates is $\nu \mathcal{U}_{0} / d$, where $\mathcal{U}_{0}$ is given by Eq. (43) and (48), which corresponds to a rather modest initial velocity of approximately $0.04 \mathrm{~m} \mathrm{~s}^{-1}$ for a bubble with no initial films to a much faster initial velocity of approximately $5 \mathrm{~m} \mathrm{~s}^{-1}$ for a bubble with initial films of (dimensional) length $L=10^{-3} \mathrm{~m}$.

\section{CONCLUSIONS}

In this paper the dynamics of a long, two-dimensional vapor bubble confined in the gap between two superheated or subcooled parallel plates was analyzed theoretically. The analysis used the approach developed by WDB to consider a situation with small gaps and large superheating or subcooling in which the mass transfer from or to the semicircular nose of the bubble is comparable to that from or to the thin liquid films on the plates. In order to permit a (semi-) analytical treatment the analysis was restricted to low Prandtl number liquids.

When both plates are superheated the bubble always expands. In this case there are two possible constant-velocity continuous-film solutions for the expansion of the bubble when $0<D<D_{\text {c }}$, namely, an unstable fast mode with velocity $U_{0}$ satisfying $U_{0}>U_{0 \mathrm{c}}$ and a stable slow mode with velocity $U_{0}$ satisfying $0<U_{0}<U_{0 \mathrm{c}}$, but none for $D>D_{\mathrm{c}}$, where the critical values $D_{\mathrm{c}}$ and $U_{0 \mathrm{c}}$ are given by Eqs. (31) and (32), respectively. The evolution of the bubble was calculated numerically for a range of values of the parameters. In particular, these calculations showed that when $0<D<D^{*}\left(\leq D_{\mathrm{c}}\right)$ the bubble eventually expands with the constant velocity of the slow mode whereas when $D>D^{*}$ it eventually expands exponentially according to Eq. (45). Numerically calculated values of $D^{*}$ are shown in Fig. 6. This behavior is qualitatively different from that in the situation investigated by WBD in which there is only a single unstable constant-velocity continuous-film solution (corresponding to the limit $D \rightarrow 0^{+}$of the present fast mode) and all bubbles always either expand indefinitely or stop in a finite time.

When both plates are subcooled the bubble always collapses to zero length at the finite time $t=t^{*}$ given by Eq. (51). This behavior is also qualitatively different from that in the situation investigated by WBD in which all bubbles always collapse to zero length asymptotically in infinite time.

When one plate is subcooled and the other plate is superheated the situation is rather more complicated. If the magnitude of the subcooling $-\Delta T_{\mathrm{u}}$ is less than that of the superheating $\Delta T_{1}$ then if $\left|\Delta T_{\mathrm{uc}}\right|<\left|\Delta T_{\mathrm{u}}\right|<\Delta T_{1}$ then a variety of

TABLE II. Values of the nondimensional parameters $P, C, D, E$, and $K$ for water and mercury corresponding to the typical physical parameter values listed in Table I.

\begin{tabular}{|c|c|c|c|c|}
\hline Nondimensional parameter & Symbol & Definition & Value for water & Value for mercury \\
\hline & & $\underline{\nu}$ & & \\
\hline \multirow[t]{2}{*}{ Prandtl number } & $P$ & $\kappa$ & 1.8 & 0.02 \\
\hline & & $\rho \nu^{2}$ & $1.4 \times 10^{-9}$ & $1.3 \times 10^{-10}$ \\
\hline \multirow[t]{2}{*}{ Capillary number } & $C$ & $\overline{\sigma d}$ & $d$ & $d$ \\
\hline & & $\rho$ & & \\
\hline \multirow[t]{2}{*}{ Density ratio } & $D$ & $\overline{\rho^{(V)}}$ & $1.6 \times 10^{3}$ & $3.25 \times 10^{3}$ \\
\hline & & $k\left|\Delta T_{1}\right|$ & & \\
\hline \multirow[t]{2}{*}{ Evaporation number } & $E$ & $\overline{\mathcal{L} \rho \nu}$ & $10^{-3}\left|\Delta T_{1}\right|$ & $0.03\left|\Delta T_{1}\right|$ \\
\hline & & $k T_{\mathrm{S}}^{3 / 2}\left(2 \pi R_{\mathrm{g}}\right)^{1 / 2}$ & $8 \times 10^{-8}$ & $6 \times 10^{-6}$ \\
\hline Kinetic parameter & $K$ & $\overline{\alpha \mathcal{L}^{2} \rho^{(V)} d}\left(\overline{M_{\mathrm{W}}}\right)$ & $\overline{\alpha d}$ & $\overline{\alpha d}$ \\
\hline
\end{tabular}


complicated behaviors (including the possibility of an unexpected "waiting time" behavior in which the bubble remains almost stationary for a finite period of time) can occur before the bubble eventually collapses to the finite length $R=R_{\infty}$ given by Eq. (54) in an infinite time, whereas if $\left|\Delta T_{\mathrm{u}}\right|$ $<\left|\Delta T_{\text {uc }}\right|$ then the bubble always expands and eventually does so exponentially according to Eq. (45). Numerically calculated values of $-\Delta T_{\mathrm{uc}}$ are shown in Fig. 12. If the magnitude of the subcooling is greater than that of the superheating then the bubble always collapses to zero length at the finite time $t=t^{*}$ given by Eq. (57). This behavior is also qualitatively different from that in the situation investigated by WBD in which all bubbles always eventually collapse to zero length asymptotically in infinite time.

\section{ACKNOWLEDGMENTS}

The present work was supported by the United Kingdom Engineering and Physical Sciences Research Council (EPSRC) Research under Grant No. GR/R74468.
[1] S. K. Wilson, S. H. Davis, and S. G. Bankoff, J. Fluid Mech. 391, 1 (1999).

[2] F. P. Bretherton, J. Fluid Mech. 10, 166 (1961).

[3] V. S. Ajaev and G. M. Homsy, J. Colloid Interface Sci. 240, 259 (2001)

[4] V. S. Ajaev and G. M. Homsy, J. Colloid Interface Sci. 244, $180(2001)$

[5] V. S. Ajaev, G. M. Homsy, and S. J. S. Morris, J. Colloid Interface Sci. 254, 346 (2002).

[6] V. S. Ajaev and G. M. Homsy, Annu. Rev. Fluid Mech. 38, 277 (2006).

[7] A. Mukherjee and S. G. Kandlikar, Microfluid. Nanofluid. 1, 137 (2005)

[8] G. Wang and P. Cheng, Int. J. Heat Mass Transfer 52, 79 (2009).

[9] S. G. Kandlikar, ASME J. Heat Transfer 126, 8 (2004).

[10] J. R. Thome, Int. J. Heat Fluid Flow 25, 128 (2004).

[11] D. B. R. Kenning, D. S. Wen, K. S. Das, and S. K. Wilson, Int. J. Heat Mass Transfer 49, 4653 (2006).

[12] J. P. Burelbach, S. G. Bankoff, and S. H. Davis, J. Fluid Mech. 195, 463 (1988).

[13] C. H. Panzarella, Ph.D. Thesis, Northwestern University, 1998 (unpublished).

[14] R. Marek and J. Straub, Int. J. Heat Mass Transfer 44, 39 (2001).

[15] www.comsol.com
[16] S. Saravanan and P. Kandaswamy, ASME J. Heat Transfer 122, 602 (2000).

[17] In the special case of equal superheating (i.e., when $\left.\Delta T_{\mathrm{u}}=\Delta T_{1}=1\right)$ the argument of the logarithm in Eq. (41) is multiplied by a factor of 2 .

[18] Note that a comparison of the present stability analysis with the corresponding analysis reported by WDB reveals that the latter is in error when $D \neq 1$. Specifically, the first term on the right hand side of their Eq. (57) [corresponding to the present Eq. (37)] should be multiplied by $U_{0}$ and so, in particular, the $D^{2 / 3}$ term in their Eq. (59) should be replaced by unity. Setting $\Delta T_{\mathrm{u}}=\Delta T_{1}$ and $U_{0}=D^{-3 / 2}$ in the present Eq. (39) recovers the corrected version of their Eq. (59) when the different definitions of $\beta$ are taken into account. Since $D$ now appears only in the corrected version of their Eq. (59) in the combination $\beta / D$ the solution for $\beta$ in this case is simply $\beta=0.7799 D$ for all values of $D$. Fortunately, the qualitative conclusions of WDB's stability analysis are not affected by this minor error.

[19] The extensive review of literature concerning the evaporation and condensation coefficients of water by Marek and Straub [14] indicates that values anywhere between 1 and $O\left(10^{-4}\right)$ are possible depending on a variety of factors including the local temperature and pressure and whether the free surface is stagnant or dynamically renewing, and so the value $\alpha=1$ used here may significantly overestimate the local mass flux. 